\title{
The effectiveness of exogenous melatonin versus transcranial magnetic stimulation on the quality of sleep, memory and mood of young adult people
}

\begin{abstract}
Melatonin is a neurohormone that is secreted in the brain and which is associated with the sleep cycle, its clinical uses have been focused on sleep disorders, as well as on the improvement of cognitive performance and people's mood. Likewise, Transcranial Magnetic Stimulation (TMS) has been used in the same areas. The objective of this research was to analyze for two weeks, in young adults with an average age of 24 (the minimum age was 18 and the maximum was 32), which of the two treatments was more effective in inducing improvements in sleep quality, memory and mood. Four groups were formed, the placebo group $(n=28)$, the sham group $(n=28)$, the melatonin group $(n=25)$ and the TMS group $(n=16)$. All groups had a pre-test evaluation of sleep quality, memory, depression and anxiety. The experimental phase lasted 2 weeks and consisted of placebo exposure, sham stimulation, melatonin consumption $(10 \mathrm{mg})$ and TMS $(128 \mathrm{~Hz})$. After this period, the post-test evaluation was carried out. The results showed that both treatments were equally effective in improving sleep quality, although TMS was more effective in improving memory and anxiety symptoms. It is inferred that both treatments are effective, although the question arises about their long-term use and the maintenance of the improvements.
\end{abstract}

Keywords: melatonin, TMS, depression, anxiety, sleep quality, memory

\author{
Volume 7 Issue 4 - 2019
}

\begin{abstract}
Sánchez-Betancourt Javier, ${ }^{1,2}$ Meza-Amaya Arturo,' Muñiz-Salazar Raquel, ${ }^{3}$ GuzmánCortes Jorge, ${ }^{2}$ Cárdenas-Bautista Dilayaxy ${ }^{3}$ 'Facultad de Ciencias Administrativas y Sociales, carrera de psicología, Unidad Valle Dorado, Universidad Autónoma de Baja California, México

2Facultad de Estudios Superiores Iztacala, Laboratorio de Neuromorfología, Universidad Nacional Autónoma de México, México

${ }^{3}$ Laboratorio de Epidemiología y Ecología Molecular, Escuela de Ciencias de la Salud, Unidad Valle Dorado, Universidad Autónoma de Baja California, México
\end{abstract}

\begin{abstract}
Correspondence: Javier Sánchez-Betancourt, Facultad de Ciencias Administrativas y Sociales, carrera de psicología Unidad Valle Dorado, Universidad Autónoma de Baja California, Ensenada, Baja California 22890, México, Tel +52 6461766600 ext I5I, Email tadeo.sanchez@uabc.edu.mx
\end{abstract}

Received: July 24, 2019 | Published: August 07, 2019
Abbreviations: TMS, transcranial magnetic stimulation

\section{Introduction}

Melatonin (N-Methyl-5-Methoxytryptamine) is a neurohormone that is synthesized in the pineal gland as well as in other organs of the body and their functions are crucial to vertebrate's life since they include the regulation of circadian rhythms by facilitating sleep and being a free radical scavenger in the brain. ${ }^{1-6}$ Moreover, the intake of exogenous melatonin has been associated with more complex functions such as being effective in improving the conditions of animals in depression/anxiety models as well as in patients with mood disorders. ${ }^{7-10}$

In addition to being effective in treating insomnia problems in older adults, ${ }^{11}$ melatonin is also effective in improving cognitive functions such as memory. ${ }^{12-15}$ Although it is not clearly known how exogenous melatonin promotes such improvement, both in humans and in laboratory animals, it has been found to promote the expression of antioxidant enzyme, ${ }^{16}$ the increase in concentration of trophic factors ${ }^{14}$ as well as increased neurogenesis in the hippocampus. ${ }^{17}$

On the other hand, it is also known that transcranial magnetic stimulation is effective in treating similar disorders in which melatonin has been effective. ${ }^{18-20}$ That is the reason why the question arises of which of the two therapies is more effective in treating both sleep disorders and cognitive functioning. Thus, the objective of this investigation was to evaluate the independent effect of the administration of exogenous melatonin and transcranial magnetic stimulation on the quality of sleep, memory and mood in young adults.

\section{Material and methods}

Participants; 100 young people from the city of Ensenada, Baja California were asked to participate in the research, which was then submitted to the ethics committee of the School of Health Sciences of the Autonomous University of Baja California. Each participant was given an informed consent where he or she was aware of the objective of the investigation and was told that it could be distributed randomly in the different experimental conditions. The average age of the participants was 24, the percentage of women and men was $36 \%$ and $64 \%$, respectively. Participants who were under psychopharmacological and/or psychological treatment were excluded from the study.

\section{Materials/equipment}

To evaluate the working memory, the computerized version of the memory span and digit span tests were applied using the PEBL platform. To measure the quality of sleep, a Pittsburgh sleep quality inventory was applied. Burns inventories were used to detect levels of depression and anxiety. The anxiety inventory has a score of 0 to 100 and the classification of minimum anxiety (0-4), limit (5-10), light (11-20), moderate (21-30), severe (41-50) and extreme (51-100). The inventory of depression has a score of 0 to 100 and is classified in the categories of non-deprecated (0-5), normal but unhappy (6-10), minimal depression (11-25), moderate depression (26-50), severe depression (51-75) and extreme depression (76-100). ${ }^{21}$

Melatonin: $10 \mathrm{mg}$ sublingual melatonin tablets from the Eurovital nutraceuticals brand were used. Participants were asked to ingest the pill a few minutes before sleeping for two consecutive weeks.

Transcranial magnetic stimulation: The portable version of the Actipulse Home-depression device was used. The stimulation protocol consisted of placing the diadem to the participants for 30 minutes a day, from Monday to Friday for two weeks. The electromagnetic impulses generated by the main unit of the stimulator are square waves with an emission frequency of approximately $128 \mathrm{Hertz}(\mathrm{Hz})$. 
Procedure: the participants were randomly distributed to one of the following groups (it was a double-blind study); 1. Placebo group $(\mathrm{n}=28)$ (this group only received strawberry flavor pills and had psychometric tests); 2. Sham group $(\mathrm{n}=28)$ (this group wore the stimulation headband and it was placed without any current and the tests were performed) 3 . Melatonin group $(\mathrm{n}=25)$ (this group received $10 \mathrm{mg}$ melatonin tablets that were ingested a few minutes before sleeping for 2 weeks, the formerly mentioned tests were applied before and after the experiment) and 4. TMS ( $\mathrm{n}=16)$ (this group received transcranial magnetic stimulation through a diadem 30 minutes a day for two weeks and the tests were also applied before and after the experiment). All groups had a pre-test evaluation of sleep quality, memory, depression and anxiety. The experimental phase lasted 2 weeks and consisted of placebo exposure, sham stimulation, melatonin consumption $(10 \mathrm{mg})$ and TMS $(128 \mathrm{~Hz})$. After this period, the post-test evaluation was carried out

\section{Statistical analysis}

The two-factor ANOVA was used where the dependent variables were quality of sleep, memory, depression and anxiety: the factors included in the analysis were "time" (that is, before and after the experimental intervention) and the "experimental condition" (that is, the group to which it belonged to). For multiple comparisons the data was analyzed using the Tukey test. The statistical program Graph Pad Pris 8 for Mac was used.

\section{Results}

In the applied quality of sleep scale, values close to 0 indicate quality of sleep while those close to 21 indicate poor quality of sleep. According to the cut-off point of the instrument, values above 5 indicate poor sleep quality. As can be seen in Figure 1, both in the placebo group and in the sham group the average quality of sleep exceeds the cut-off point and remained similar in the pre-test and post-test phase of the experiment. In the melatonin and TMS groups, an improvement in sleep quality was observed in both groups, with both of them approaching a 5. The two factor ANOVA showed that time, experimental condition as well as interaction of both groups were significant $[\mathrm{F}(3,80)=7,285, \mathrm{p}<0.01]$. The analysis of multiple comparisons confirmed that the melatonin and TMS groups showed the reported improvement in sleep quality.

\section{Sleep Quality}

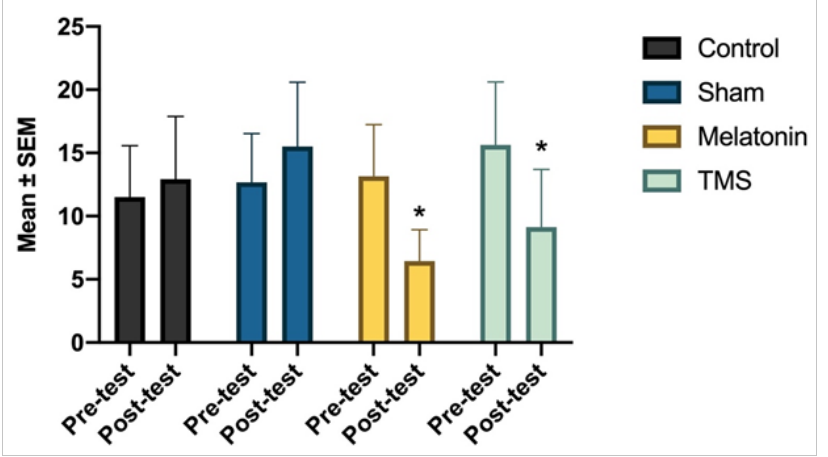

Figure I Shows the quality of sleep in the four groups before and after receiving the treatments; In the melatonin and TMS groups there was an improvement in sleep quality. The interaction between the "time" factor and "experimental condition" was significant in these groups $[\mathrm{F}(3,80)=7,285]$. * Versus control, $\mathrm{p}<0.00$ I

Regarding the execution of the memory tests, it can be seen in Figure 2 that in the memory span test the placebo, sham and melatonin groups had similar averages in the pre-test and post-test phases; however, the TMS group showed significant differences in the posttest phase $[\mathrm{F}(3,60)=6,92, \mathrm{p}<0.01]$, which indicates an increase in the ability to remember images. In the digit span test, no significant differences were found due to the time factor, experimental condition or the interaction of both, however, an increase in the average of items remembered can be seen (see Figure 3). For example, the Melatonin group had an average of 5.5 and 6.5 of items in the pre-test and posttest evaluations, respectively; on the other hand, the TMS group had an average of 5.8 and 8 items before and after stimulation, respectively. Memory span

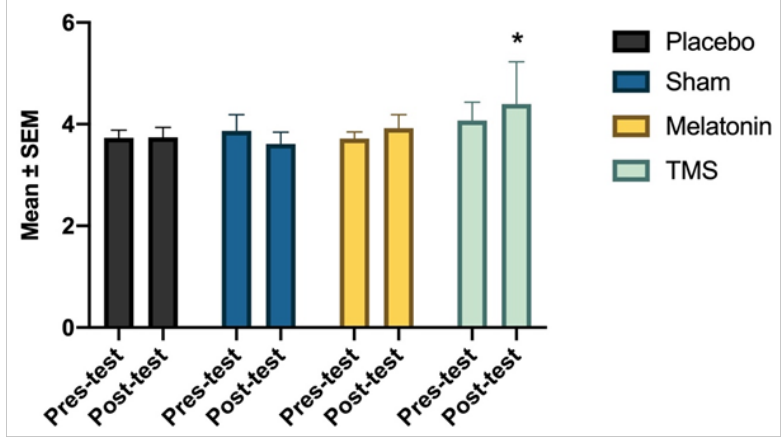

Figure 2 Shows the average of correct answers in the Mspan test in the four groups before and after receiving the treatment. While in the placebo, sham and melatonin groups there are no changes in the pre-test and post-test evaluations in the TMS group, the two-way ANOVA revealed a significant effect of the "experimental condition" factor $[F(3,60)=6,922]$. * Versus control, $P$ $<0.00$ l

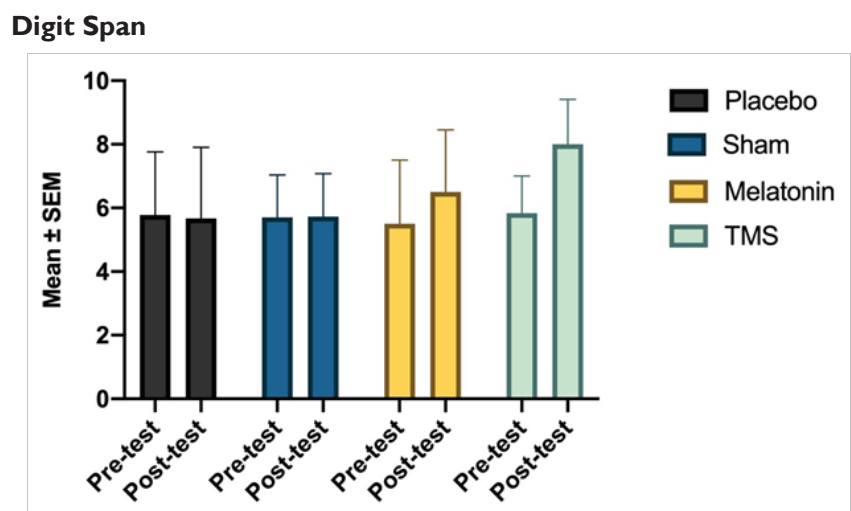

Figure 3 Shows the average number of correct answers obtained in the Dspan test in the four groups before and after receiving the treatments. Statistical analyzes did not reveal significant changes after treatments. However, it can be seen in the experimental groups a tendency to improve in the execution of said test. $*$ Versus control, $\mathrm{p}<0.00$

The average levels of depression found in the pre-test phase in the four groups were similar and according to the interpretation of the Burns depression inventory corresponded to the levels of moderate depression (Figure 4). In the post-test phase, this trend was maintained with the exception of the melatonin group, where there was a combined effect of the "time" factor and "experimental condition" to significantly reduce depression values $[F(3,98)=3,103, p<0.01]$. The average levels of depression in this group after melatonin treatment corresponded to those of minimal depression.

Anxiety in the placebo and sham groups was similar in the pre-test and post-test evaluations (Figure 5). According to the classification of the Burns anxiety inventory in both groups, it was detected that the average score corresponded to a moderate level of anxiety. In the case of the melatonin group, the pre-test evaluation showed moderate 
anxiety and in the post-test evaluation the participants' scores corresponded to those of light anxiety. Statistical analysis showed that the experimental condition factor had a significant reduction in anxiety in the TMS group $[\mathrm{F}(3,96)=4,23, \mathrm{p}<0.01]$

\section{Depression}

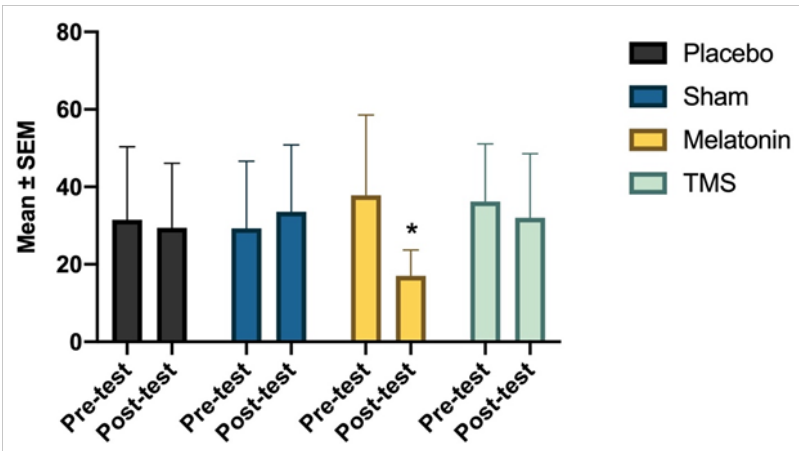

Figure 4 Depression levels in the melatonin group significantly decreased after the two week treatment $[\mathrm{F}(3,98)=3,103]$. *Versus control, $\mathrm{p}<0.00$ I

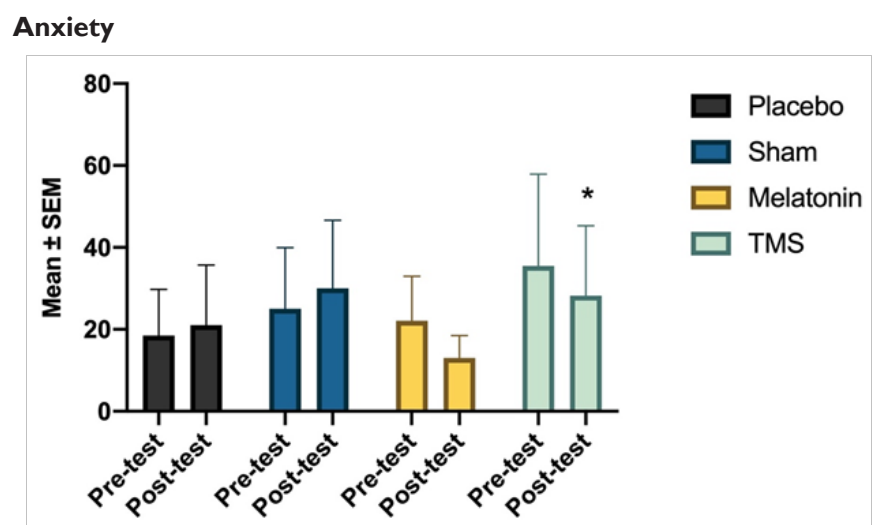

Figure 5 Shows the anxiety levels before and after the experimental phase.As can be seen, anxiety levels had a decrease in the TMS group; Statistical analyzes showed that the experimental condition factor had a significant effect on $[F(3$, 96) $=4,227]$. * Versus control, $\mathrm{p}<0.001$

\section{Discussion}

Sleep quality is a condition that was affected in all participants of this research. However, the melatonin treatment as well as the magnetic stimulation treatment was effective in improving the quality of sleep as reported by the participants. This data is consistent with reports where exogenous melatonin is useful and widely used to treat sleep disorders. ${ }^{22}$ Thus, for example, there is evidence where relatively low doses of melatonin ( $1 \mathrm{mg} /$ day) administered for 5 weeks (of which 2 received placebo) were effective in reducing the difficulty of waking up, reducing sleep during school hours and increasing sleep time in adolescents whose ages ranged from 14 to $19 .{ }^{23}$ Our data also agreed with studies where patients who were in a treatment against heroin addiction and who underwent a $10 \mathrm{~Hz}$ stimulation protocol for six weeks reported a significant improvement in sleep quality. ${ }^{24}$ While melatonin was more effective in improving sleep quality, statistical analyzes did not show that one treatment was more effective than the other. These results could be explained because melatonin has the natural function of regulating sleep cycles, increasing the REM sleep cycle and decreasing sleep latency. ${ }^{2}$ In the case of TMS, it has been shown that it induces the propagation of slow waves, similar to those of the deep sleep phase. ${ }^{25}$

Memory improvement can be clearly seen in the memory span test in the group that received TMS for two weeks. These results agree with reports where TMS in the motor cortex is able to improve the learning of motor sequences in 22 year olds. ${ }^{26}$ Similarly, they agree with memory improvement in mice exposed to microgravity conditions (which causes cognitive damage); in that case, $15 \mathrm{~Hz}$ TMS was applied for 14 consecutive days. ${ }^{27}$ In this investigation, memory improvement was associated with an increase in dendritic spine density of the dentate gyrus of the hippocampus, as well as an increase in the expression of postsynaptic proteins NR2A, NR2B, PSD95 (associated with memory formation ) as well as an increase in BNDF/ TrkB growth factors. On the other hand, although melatonin was not able to significantly improve performance in memory tests, although if there is a tendency to increase the average number of successes in both tests and it seems that the effects on this function are observed with more days of treatment, at least for two more weeks. ${ }^{28}$

The depression variable was the one that got benefited the most by the melatonin treatment. This is consistent with research in which mice that received the $10 \mathrm{mg} / \mathrm{k}$ intraperitoneal injection of melatonin and that had been pretreated with liposaccharides had a reduction in depressive symptoms induced by such drugs. This improvement was associated with the increase in glutathione antioxidant enzyme, increase in BNDF and decrease in TNF-a in the hippocampus. ${ }^{29}$ On the other hand, these results are consistent with the fact that the administration of $10 \mathrm{mg} / \mathrm{k}$ melatonin in rats was able to reverse depressive symptoms induced by continuous stress. Neurochemical analyzes confirmed that this improvement was related to an increase in norepinephrine levels in the hippocampus. ${ }^{30}$ Furthermore, our results agree with preclinical studies where melatonin has shown antidepressant properties. Although statistical analyzes did not show a reduction in depression levels in the TMS group if a tendency to decrease can be seen, which would be consistent with reports of the effectiveness of this treatment for depression. ${ }^{31,32}$

Anxiety showed high values in all the groups evaluated and decreased with experimental conditions. This data is consistent with reports where TMS was effective for the treatment of anxiety disorders. ${ }^{2,3}$ It should be noted that the group that had higher levels of anxiety before the experiment was that of TMS and after that treatment the anxiety levels decreased significantly, so it is likely that TMS use has higher anxiolytic properties than that of melatonin's.

\section{Conclusion}

After two weeks of treatment, both melatonin and TMS were effective in improving the sleep quality of young adults. TMS was more effective than melatonin for relieving anxiety symptoms and for improving memory test scores. On the other hand, the melatonin treatment was more effective in reducing the symptoms of depression. However, there is still the question of knowing how the effectiveness of such treatments would be long-term (one or two months) and knowing if these effects are maintained after the end of the treatment.

\section{Funding details}

My research project was partially or fully sponsored by (PRODEP) with grant number (UABC-PTC-691).

\section{Acknowledgments}

None. 


\section{Conflicts of interest}

The authors declare no conflict of interest.

\section{References}

1. Vanecek J. Cellular Mechanisms of Melatonin Action. Physiol Rev. 1998;78(3):687-721.

2. Boutin JA, Audinot V, Ferry G, et al. Molecular tools to study melatonin pathways and actions. Trends Pharmacol Sci. 2005;26(8):412-419.

3. Leon J, Acuña-Castroviejo D, Sainz RM, et al. Melatonin and mitochondrial function. Life Sci. 2004;75(7):765-790.

4. Rebai R, Jasmin L, Boudah A. The antidepressant effect of melatonin and fluoxetine in diabetic rats is associated with a reduction of the oxidative stress in the prefrontal and hippocampal cortices. Brain Res Bull. 2017; 134:142-150.

5. Kundurovic Z, Sofic E. The effects of exogenous melatonin on the morphology of thyrocytes in pinealectomized and irradiated rats. $J$ Neural Transm. 2006;113(1):49-58.

6. Sharif R, Aghsami M, Gharghabi M, et al. Melatonin reverses H-89 induced spatial memory deficit: Involvement of oxidative stress and mitochondrial function. Behav Brain Res. 2017;316:115-124.

7. Haridas S, Kumar M, Manda K. Melatonin ameliorates chronic mild stress induced behavioral dysfunctions in mice. Physiol Behav. 2013;119:201-207.

8. Sun X, Wang M, Wang Y, et al. Melatonin produces a rapid onset and prolonged efficacy in reducing depression-like behaviors in adult rats exposed to chronic unpredictable mild stress. Neurosci Lett. 2017;642:129-135.

9. Khanna P, Maitra S, Baidya D. Melatonin in perioperative medicine: Current perspective. Saudi J Anaesth. 2013;7(3):315-321.

10. Tchekalarova J, Stoynova T, Ilieva K, et al. Agomelatine treatment corrects symptoms of depression and anxiety by restoring the disrupted melatonin circadian rhythms of rats exposed to chronic constant light Pharmacol Biochem Behav. 2018;171:1-9.

11. Garfinkel D, Laudon M, Nof D, et al. Improvement of sleep quality in elderly people by controlled-release melatonin. Lancet. 1995;346(8974):541-544.

12. Brusco LI, Márquez M, Cardinali DP. Monozygotic twins with alzheimer's disease treated with melatonin: Case report. J Pineal Res. 1998;25(4):260-263.

13. Jean-Louis G, Von Gizycki H, Zizi F. Melatonin effects on sleep, mood, and cognition in elderly with mild cognitive impairment. J Pineal Res. 1998; 25(3):177-183.

14. Chen BH, Park JH, Lee TK, et al. Melatonin attenuates scopolamine-induced cognitive impairment via protecting against demyelination through BDNF-TrkB signaling in the mouse dentate gyrus. Chem Biol Interact. 2018;285:8-13.

15. Alzoubi KH, Mayyas FA, Mahafzah R, et al. Melatonin prevents memory impairment induced by high-fat diet: Role of oxidative stress. Behav Brain Res. 2018;336:93-98.

16. Mayo JC, Sainz RM, Antolín I, et al. Melatonin regulation of antioxidant enzyme gene expression. Cell Mol Life Sci. 2002;59(10):1706-1713.
17. Ruksee N, Tongjaroenbuangam W, Mahanam T, et al. Melatonin pretreatment prevented the effect of dexamethasone negative alterations on behavior and hippocampal neurogenesis in the mouse brain. J Steroid Biochem Mol Biol. 2014;143:72-80.

18. Rodriguez DA, Morelos SE, Torres MA, et al. Effect of repetitive transcraneal magnetic stimulation on aggressive impulsive behavior in subjects with bpd in a of social exclusion paradigm. Brain Stimul. 2019;12(2):512.

19. Koch G, Bonnì S, Pellicciari MC, et al. Transcranial magnetic stimulation of the precuneus enhances memory and neural activity in prodromal Alzheimer's disease. Neuroimage. 2018;169:302-311.

20. Lin J, Liu X, Li H, et al. Chronic repetitive transcranial magnetic stimulation (rTMS)on sleeping quality and mood status in drug dependent male inpatients during abstinence. Sleep Med. 2019;58:7-12.

21. Wei S, Smits MG, Tang X, et al. Efficacy and safety of melatonin for sleep onset insomnia in children and adolescents: a meta-analysis of randomized controlled trials. Sleep Medicine. 2019.

22. Burns DD. The Feeling Good Handbook. Rev. ed. New York, USA: Plume; 1999.

23. Eckerberg B, Lowden A, Nagai R, Åkerstedt T. Melatonin Treatment Effects on Adolescent Students' Sleep Timing and Sleepiness in a Placebo-Controlled Crossover Study. Chronobiol Int. 2012;29(9):1239-1248.

24. Yang LL, Zhao D, Kong LL, et al. High-frequency repetitive transcranial magnetic stimulation (rTMS) improves neurocognitive function in bipolar disorder. J Affect Disord. 2019;246:851-856.

25. Massimini M, Ferrarelli F, Esser SK, et al. Triggering sleep slow waves by transcranial magnetic stimulation. Proc Natl Acad Sci U S A. 2007;104(20):8496-8501.

26. Nojima I, Watanabe T, Gyoda T, et al. Transcranial static magnetic stimulation over the primary motor cortex alters sequential implicit motor learning. Neurosci Lett. 2019;696:33-37.

27. Zhai B, Fu J, Xiang S, et al. Repetitive transcranial magnetic stimulation ameliorates recognition memory impairment induced by hindlimb unloading in mice associated with BDNF/TrkB signaling. Neurosci Res. 2019;pii:S0168-0102(18)30725-9.

28. Sánchez-Betancourt J, Avila-Costa MR, Meza-amaya, et al. Acute effect of exogenous melatonin on cognitive functions in young adults who consume alcohol. Archivos Venezolanos de Farmacología y tepaoeútica. 2018;37(5):2-5.

29. Taniguti EH, Ferreira YS, Stupp IJV, et al. Neuroprotective effect of melatonin against lipopolysaccharide-induced depressive-like behavior in mice. Physiol Behav. 2018;188:270-275.

30. Stefanovic B, Spasojevic N, Jovanovic P. Melatonin mediated antidepressant-like effect in the hippocampus of chronic stress-induced depression rats : Regulating vesicular monoamine transporter 2 and monoamine oxidase A levels. Eur Neuropsychopharmacol. 2016;26(10):1629-1637.

31. Chung SW, Hoy KE, Fitzgerald PB. Theta-burst stimulation: a new form if TMS treatment for depression? Depress Anxiety. 2015;32(3):182-192.

32. Fitzgerald PB, Hoy KE, Elliot D, et al. Accelerated repetitive transcranial magnetic stimulation in the treatment of depression. Neuropsychopharmacology. 2018;43(7):1565-1572. 